\title{
Modified Digital Backpropagation Accounting for Polarization-Mode Dispersion
}

\author{
Cristian B. Czegledi ${ }^{1}$, Gabriele Liga ${ }^{2}$, Domaniç Lavery², Magnus Karlsson ${ }^{3}$, \\ Erik Agrell $^{1}$, Seb J. Savory ${ }^{4}$, and Polina Bayvel ${ }^{2}$ \\ ${ }^{1}$ Dept. of Signals and Systems, Chalmers University of Technology, Gothenburg, SE-412 96, Sweden \\ ${ }^{2}$ Dept. of Electronic and Electrical Engineering, University College London, London, WC1E 7JE, U.K. \\ ${ }^{3}$ Dept. of Microtechnology and Nanoscience, Chalmers University of Technology, Gothenburg, SE-412 96, Sweden \\ ${ }^{4}$ Dept. of Engineering, Electrical Engineering Division, University of Cambridge, Cambridge, CB3 OFA, U.K. \\ czegledi@chalmers.se
}

\begin{abstract}
We propose a modified DBP algorithm accounting for PMD. The accumulated PMD at the receiver is factorized into several PMD sections, and inserted into the DBP routine to distributively compensate for PMD, outperforming the conventional approach by $1.1 \mathrm{~dB}$ in SNR.
\end{abstract}

OCIS codes: 060.0060 Fiber optics and optical communications, 060.1660 Coherent communications.

\section{Introduction}

Digital signal processing (DSP) effectively mitigates linear fiber impairments, such as chromatic dispersion (CD) and polarization-mode dispersion (PMD), whereas the intensity-dependent Kerr nonlinearity is suggested to be the ultimate obstacle of the optical fiber capacity. Due to the nonlinear effects, there exists an optimal (effective) signal-to-noise ratio (SNR) point at the compromise between additive noise and nonlinear interference where it is recommended to operate. However, various nonlinear interference mitigation techniques are currently under investigation, among which digital backpropagation (DBP) has proved to be promising [1]. DBP compensates for the deterministic fiber nonlinear impairments by solving the nonlinear propagation equation using the split-step Fourier (SSF) method and backpropagating the received optical field with inverted channel parameters. It is believed that the deterministic nonlinear signal-signal interactions are completely removed using DBP and that the performance of a fiber-optical system is limited by the uncompensated stochastic effects, such as amplified spontaneous emission noise, which leads to signal-noise interactions, and PMD leading to polarization-dependent nonlinear interactions [2-4].

PMD introduces a frequency-dependent delay that accumulates as a random-walk-like process along the fiber length and it is usually compensated for at the receiver after DBP. When applying DBP, the entire reverse propagation is performed with the accumulated PMD over the entire link; therefore the nonlinear compensation is mismatched and its accuracy degrades with the backpropagated distance. In order to avoid this effect, PMD should be compensated for as it naturally occurs, i.e., in a distributed fashion along the link, rather than doing it at once after DBP. It was shown numerically that compensating for PMD on a per span-basis decreases its impact on DBP significantly [5]. However, this approach requires a priori PMD knowledge for every span, which is challenging to realize.

Recently, a modified DBP algorithm that takes into account PMD was proposed in [6], where an appropriate amount of differential group delay (DGD) at the link principal states of polarization (PSP) is introduced after each span, such that the accumulation of DGD in the forward propagation is reversed. In [7], we recently proposed a modified DBP method that reverses the PMD effects in the backward propagation by passing the reverse propagated signal through PMD sections. Unlike [6] where the DGD is subtracted along the same PSP every time in the backward propagation, in [7] the PSPs of the backward PMD sections are different and are aligned using an optimization algorithm such that the Jones matrix modeling the total backward PMD equals the inverse of the Jones matrix modeling the PMD occurring in the forward propagation. However, low-complexity DBP implementations (one step per span or less) are marginally affected by polarization effects, since the nonlinear signal-signal interactions are not entirely removed and dominate the achievable SNR [8].

In this paper, a DBP algorithm accounting for PMD is proposed where the PMD sections are computed analytically using a first-order linearization approach, yielding in better performance at lower complexity.

\section{Proposed Algorithm}

The schematic diagram of the proposed algorithm is shown in Fig. 1. As can be seen, the DBP algorithm is connected through a feedback loop with the channel equalizer, such as the constant/multiple modulus algorithm (CMA/MMA), compensating for the PMD. The equalizer estimates the inverse of the accumulated PMD over the link, which can be modeled by a frequency-dependent Jones matrix $\mathbf{J}(f)$. The matrix $\mathbf{J}(f)$ is fed back to the DBP algorithm, which divides $\mathbf{J}(f)$ into several sections that are spread evenly in the DBP routine. These sections are calculated by first approximating the matrix $\mathbf{J}(f)$ to its first order at $f_{0}$ as $\mathbf{J}(f) \approx \mathbf{J}\left(f_{0}\right)+\left(f-f_{0}\right) \mathbf{J}^{\prime}\left(f_{0}\right)$, where $(\cdot)^{\prime}$ denotes the first 


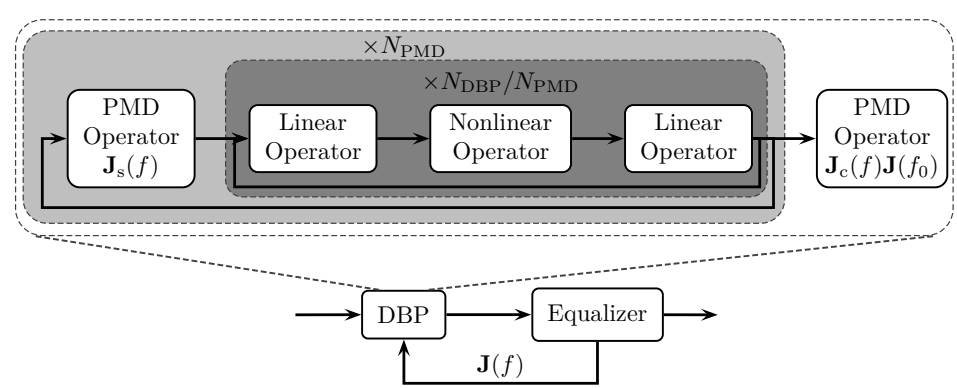

Fig. 1. Schematic of the proposed DBP method, where $N_{\mathrm{DBP}}$ is the number of steps used by the DBP algorithm over the entire link and the equalizer is a conventional channel equalizer such as the CMA or MMA. For brevity, the blocks modeling the amplifiers and attenuation are not shown.

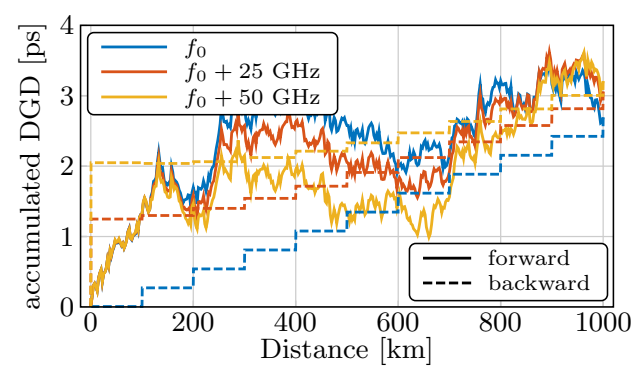

Fig. 2. An example of the evolution of the DGD at different frequencies versus distance in the forward and backward propagation. The PMD parameter is $0.1 \mathrm{ps} / \sqrt{\mathrm{km}}$ and $N_{\mathrm{PMD}}=10$.

derivative with respect to frequency. Thereafter, based on the approximation $\mathbf{I}+\mathbf{A} \approx \exp (\mathbf{A})$ for $\mathbf{A} \in \mathbb{C}^{2}, \mathbf{J}(f)$ can be expressed as $\mathbf{J}(f) \approx \mathbf{J}\left(f_{0}\right) \mathbf{J}_{\mathrm{s}}(f)^{N_{\mathrm{PMD}}}$, where $\mathbf{J}_{\mathrm{s}}(f)$ is defined in (1) and $(\cdot)^{-1}$ denotes the inverse operator.

$$
\mathbf{J}_{\mathrm{s}}(f)=\exp \left(\frac{\left(f-f_{0}\right) \mathbf{J}^{-1}\left(f_{0}\right) \mathbf{J}^{\prime}\left(f_{0}\right)}{N_{\mathrm{PMD}}}\right)
$$

$$
\mathbf{J}_{\mathrm{c}}(f)=\mathbf{J}(f)\left(\mathbf{J}\left(f_{0}\right) \mathbf{J}_{\mathrm{s}}(f)^{N_{\mathrm{PMD}}}\right)^{-1}
$$

The operator $\mathbf{J}_{\mathrm{s}}(f)$ is nested in the DBP procedure and applied $N_{\text {PMD }}$ times to distributively compensate for PMD in the reverse propagation, whereas $\mathbf{J}\left(f_{0}\right)$ is applied only once after DBP. The involved approximations in deriving $\mathbf{J}_{\mathrm{s}}(f)$ hold tightly around $f_{0}$, which is chosen to be in the middle of the signal bandwidth, but diverge as $f$ deviates from $f_{0}$. To correct for these approximations, a correction matrix $\mathbf{J}_{\mathbf{c}}(f)$ defined in (2) is applied after DBP and $\mathbf{J}\left(f_{0}\right)$, such that $\mathbf{J}(f)=\mathbf{J}_{\mathrm{c}}(f) \mathbf{J}\left(f_{0}\right) \mathbf{J}_{\mathrm{S}}(f)^{N_{\mathrm{PMD}}}$. In [7], the PMD operators used in the backward propagation are calculated using an optimization algorithm, which is more demanding than calculating (1) and (2).

Fig. 1 also illustrates the internal structure of the proposed DBP method. The conventional DBP algorithm is confined in the dark gray box, whereas the two extra operators $\mathbf{J}_{\mathrm{s}}(f), \mathbf{J}_{\mathrm{c}}(f) \mathbf{J}\left(f_{0}\right)$ are added to account for PMD. Initially, the DBP algorithm operates as in the conventional approach, without compensation for PMD, and $\mathbf{J}(f)$ is estimated by the equalizer and fed back to DBP, after which PMD will be compensated for in the DBP routine. Since PMD is a time-varying stochastic process, the residual PMD after DBP due to temporal variations is mitigated by the equalizer and $\mathbf{J}(f)$ has to be periodically updated to account for this residue.

Fig. 2 illustrates an example of the evolution of the accumulated DGD in the forward propagation and in the backward propagation applying (1) and (2) to compensate for PMD. As can be seen, different frequency components accumulate different amounts of DGD in the forward propagation in a random-walk-like fashion. In the backward propagation, the $\mathbf{J}_{\mathrm{s}}(f)$ operator is applied at every multiple of $100 \mathrm{~km}$ starting from distance $1000 \mathrm{~km}$, whereas $\mathbf{J}_{\mathrm{c}}(f) \mathbf{J}\left(f_{0}\right)$ is applied last, at distance $0 \mathrm{~km}$. The DGD at $f_{0}$, where the first order approximation is performed, decreases after every $\mathbf{J}_{\mathrm{s}}(f)$ operation with a constant step equal to $1 / N_{\mathrm{PMD}}$ of the total DGD at $1000 \mathrm{~km}$. On the other hand, due to the approximations involved in deriving $\mathbf{J}_{\mathrm{s}}(f)$, the DGDs at the other two frequencies do not decrease to 0 . Since the approximations are tighter around $f_{0}$, the DGD at $f_{0}+25 \mathrm{GHz}$ has a lower residual DGD at $0 \mathrm{~km}$ compared to the one at $f_{0}+50 \mathrm{GHz}$. This residual DGD is corrected by applying $\mathbf{J}_{\mathrm{c}}(f)$ at $0 \mathrm{~km}$. This operation has no impact on the DGD at $f_{0}$, since there is no approximation in this case and the accumulated DGD is successfully removed already at $100 \mathrm{~km}$.

\section{Simulation Setup}

We study through numerical simulations a point-to-point transmission link consisting of an ideal transmitter and coherent receiver, and 10 spans of $100 \mathrm{~km}$ standard single-mode fiber with one erbium-doped fiber amplifier per span compensating for the exact span loss and having a noise figure of $4.5 \mathrm{~dB}$. The transmitted signal consists of a polarizationmultiplexed 16-ary quadrature amplitude modulated channel at 50 Gbaud shaped using a root-raised cosine (RRC) pulse with roll-off factor 0.01. The signal propagation was simulated by solving the Manakov-PMD equation [9] using the SSF approach with steps of $0.1 \mathrm{~km}$. PMD was emulated at every SSF step consisting of a polarization scrambler, which uniformly [10] scatters the state of polarization, and a retardation plate. The DGD introduced by each retardation plate was Gaussian distributed with mean $\Delta \tau_{p}$ and standard deviation $\Delta \tau_{p} / 5$ [11]. In order to capture the stochastic nature of PMD, 120 fiber realizations were simulated for each set of parameters. We consider two receiver DSP setups: i) conventional DBP followed by a linear PMD equalizer, and ii) modified DBP described in the previous section. DBP is performed with the same number of SSF steps as in the forward propagation and is followed by an ideal matched RRC filter applied to the signal, after which the SNR is estimated by comparing the transmitted and received symbols.

\section{Results and Discussion}

Fig. 3a shows the achieved performance obtained for a PMD parameter of $0.1 \mathrm{ps} / \sqrt{\mathrm{km}}$, resulting in $3.16 \mathrm{ps}$ average DGD. The maximum SNR obtained in this setup compensating only for CD and PMD is $18.5 \mathrm{~dB}$, not shown in the 


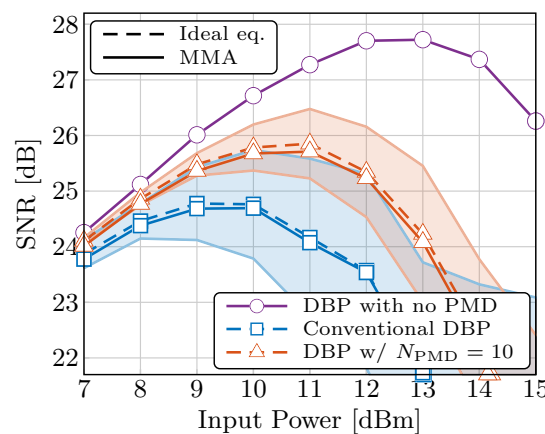

(a)

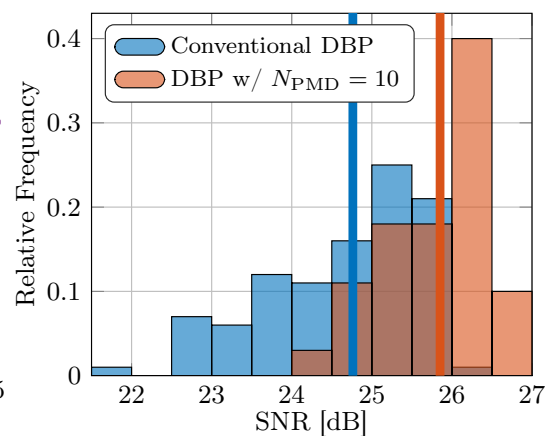

(b)

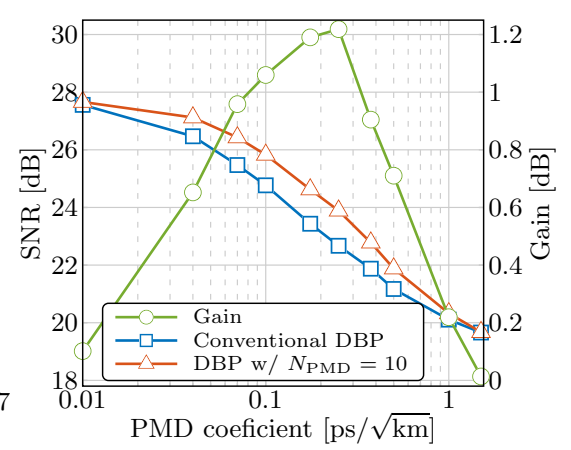

(c)

Fig. 3. (a) Average SNR versus input power. The shaded areas represent the standard deviation. (b) Histogram of the SNRs obtained by DBP and the proposed modification. The vertical thin bars represent the mean values. (c) Average optimum SNR and SNR gain versus the fiber PMD parameter obtained at the optimum input power for each scenario.

figure for conciseness. As can be seen, the performance of conventional DBP degrades in the presence of PMD by $\sim 3 \mathrm{~dB}$ and the modified DBP scheme improves the SNR by $1.1 \mathrm{~dB}$ compared to the conventional approach. In [7], a gain of only 0.7 was obtained in the same simulation setup. However, a more comprehensive study including [6,7] is required to investigate which of three methods offers the best performance for various simulation setups and channel conditions. In the same figure, we compare the performance achieved by an ideal equalizer with results based on the blind MMA with 31 taps. As can seen, the MMA induces a penalty of $\sim 0.1 \mathrm{~dB}$, which is approximately constant over the entire range of input power and is the same for both DBP schemes.

Fig. 3b illustrates the histogram of the achieved SNRs with the two DBP schemes at the optimum input power of 10 and $11 \mathrm{dBm}$, respectively, for different PMD realizations with a PMD parameter of $0.1 \mathrm{ps} / \sqrt{\mathrm{km}}$. As can be seen, the SNR range of the proposed scheme is narrower and the worst/best case is $2.5 / 0.5 \mathrm{~dB}$ better than with the conventional DBP, thus improving the required SNR margin.

Fig. 3c shows the average performance of the two schemes as a function of the PMD parameter at the optimal input power for each case. As the PMD parameter increases, the achieved SNR by both schemes degrades significantly from $27.6 \mathrm{~dB}$ down to $19.6 \mathrm{~dB}$ when the PMD coefficient is $1.5 \mathrm{ps} / \sqrt{\mathrm{km}}$. However, the proposed DBP method provides gains greater than $0.8 \mathrm{~dB}$ over the range of $0.04-0.4 \mathrm{ps} / \sqrt{\mathrm{km}} \mathrm{PMD}$ coefficient, which covers most of the modern optical fibers. The peak gain of $1.2 \mathrm{~dB}$ is achieved for $0.25 \mathrm{ps} / \sqrt{\mathrm{km}}$, after which the gain declines and becomes 0.1 $\mathrm{dB}$ at $1.5 \mathrm{ps} / \sqrt{\mathrm{km}}$. At low PMD parameters, PMD has a smaller impact on DBP, hence the small gains obtained by the proposed algorithm, whereas at high PMD parameters, it is likely that the gain deteriorates due to the approximations used to compute $\mathbf{J}_{\mathrm{s}}(f)$, which become less accurate.

\section{Conclusions}

We presented a simple modification of the DBP algorithm to blindly reverse the PMD effects in the backward propagation. The algorithm has been proved to work with PMD information obtained from the blind MMA equalizer and provides SNR gains of $1.1 \mathrm{~dB}$ for a $1000 \mathrm{~km}$ link with $0.1 \mathrm{ps} / \sqrt{\mathrm{km}}$ PMD parameter.

\section{References}

1. E. Ip, "Nonlinear compensation using backpropagation for polarization-multiplexed transmission," J. Lightw. Technol. 28, 939-951 (2010).

2. G. Gao et al., "Influence of PMD on fiber nonlinearity compensation using digital back propagation," Opt. Express 20, 14,406-14,418 (2012).

3. K. Goroshko et al., "Fundamental limitations of digital back propagation due to polarization mode dispersion," in Proc. ACP, (Hong Kong, China, 2015), p. ASu3F.5.

4. G. Liga et al., "Ultra-wideband nonlinearity compensation performance in the presence of PMD," in Proc. ECOC, (Düsseldorf, Germany, 2016), pp. 794-796.

5. F. Yaman and G. Li, "Nonlinear impairment compensation for polarization-division multiplexed WDM transmission using digital backward propagation," IEEE Photon. J. 2, 816-832 (2010).

6. K. Goroshko et al., "Overcoming performance limitations of digital back propagation due to polarization mode dispersion," in Proc. ICTON, (Trento, Italy, 2016), p. Mo.B1.4.

7. C. B. Czegledi et al., "Polarization-mode dispersion aware digital backpropagation," in Proc. ECOC, (Düsseldorf, Germany, 2016), pp. 1091-1093.

8. I. Fernandez de Jauregui Ruiz et al., "Impact of polarization mode dispersion on digital nonlinear compensation algorithms in dispersion unmanaged systems," in Proc. OFC, (Anaheim, CA, 2016), p. Th3D.3.

9. D. Marcuse et al., "Application of the Manakov-PMD equation to studies of signal propagation in optical fibers with randomly varying birefringence," J. Lightw. Technol. 15, 1735-1746 (1997).

10. C. B. Czegledi et al., "Polarization drift channel model for coherent fibre-optic systems," Sci. Rep. 6, 21217 (2016).

11. C. H. Prola et al., "PMD emulators and signal distortion in $2.48-\mathrm{Gb} / \mathrm{s}$ IM-DD lightwave systems," IEEE Photon. Technol. Lett. 9, 842-844 (1997). 